International Journal of Navigation and Port Research

Vol.30, No.4 pp. 309 315, 2007 (ISSN-1598-5725)

\title{
A Study on Mariners' Standard Behavior for Collision Avoidance (2) - A proposal of modeling method for collision avoidance based on human factors -
}

\author{
Jung-Sun Park* ·Hiroaki Kobayashi** + ' Byeong-Deok Yea*** \\ * Department of Ship Operation Systems Engineering, Korea Maritime University, Busan 606-791, Republic. of Korea \\ ** Department of Applied Marine Environmental Studies, Tokyo University of Marine Science and Technology, Tokyo 108-8477, Japan \\ *** Department of Ship Operation Systems Engineering, Korea Maritime University, Busan 606-791, Republic. of Korea
}

\begin{abstract}
We've investigated the characteristics on mariner's behavior in the collision situation through a full-mission ship handling simulator and considered that it's necessary to model the standard avoiding behavior of mariners in order to apply the obtained results more widely and effectively. Thus we described the contents of standard avoiding behavior taken by mariners in the collision situation and established the concept of the standard model based on human factors for collision avoidance in a previous study. As a following study, this paper is to propose the method of modeling on mariners' standard behavior for collision avoidance by analyzing the contents of mariner's information processing and the related factors using regression analysis. As a result, we confirmed the influence of relating factors to avoiding behavior in mariner's deciding decisions and proposed the modeling method of mariners' standard behavior for collision avoidance with a example of recognition model.
\end{abstract}

Key words : Modeling, Human factors, Mariners, Standard behavior, Collision avoidance, Information processing, Mission, Task and judgment, Information

\section{Introduction}

We've investigated mariner's characteristics in the situation of collision avoidance through a full-mission ship handling simulator and established the concept of standard model on mariners' behavior for collision avoidance (Park et al., 2003; Kobayashi, 2004; Kobayashi, 2005a). In the study, we defined that mariner's avoiding behavior is a continuous sequence of information processing of a human in ship handling for collision avoidance. We described the procedure of mariner's avoiding behavior for collision avoidance and categorized the procedure into six processes that are detection, identification, recognition, plan, execution and return according to the purpose of information processing to attain. And we explained the each process focused on four factors that are one mission, the mission-related information processing, necessary information for information processing and the available way to obtain necessary information.

In this paper, as a following study, we intended to propose the method to model mariner's behavior by analyzing the contents of mariner's information processing and the related factors. Thus we formulated the relation between mariner's behavior and the related factors in recognition process by using regression analysis. As a result, the modeling method on standard avoiding behavior is suggested with giving an example of recognition process with the obtained result from international collaborative research on human factors (Kobayashi, 2005b) in this paper.

\section{Standard Avoiding Behavior}

We categorized mariner's avoiding behavior in the situation of collision avoidance into six processes as mentioned above and explained each process with three factors, which are <Mission>, <Information processing: Task and Judgment $>$ and <Relating information $>$. In this section, the concept on the relation between information, mariner's information processing and action to avoid collision in navigational environment is introduced.

Fig. 1 shows the relation between input and output in mechanical system, which gives the specific output responding to the contents of input (Kobayashi, 2003). By using the relation between the input and output in the mechanical system of Fig. 1, the model of mariner's behavior can be expressed by the relation between information $\left(X: x_{1}, x_{2}, x_{3}, \cdots, x_{n}\right)$, mariner's information processing ( $\mathrm{F}$ ) and action ( $\mathrm{Y}$ ) as shown in Fig. 2 (Park et al., 2007). In other words, mariner's action (Y) is resulted

\footnotetext{
* Jung-Sun Park, goghpark@hanmail.net, 051) 410-4243

** Kobayasi@e.kaiyodai.ac.jp, (03) 5245-7398

+ Corresponding Author : byea@hhu.ac.kr, 051) 410-4243
} 
from their information processing $(F)$ based on the given information $\left(\mathrm{X}: \mathrm{x}_{1}, \mathrm{x}_{2}, \mathrm{x}_{3}, \ldots, \mathrm{x}_{\mathrm{n}}\right.$ ), which can be expressed by following equation,

$$
Y=F(X)=F\left(x_{1}, x_{2}, x_{3}, \ldots, x_{n}\right)
$$

Thus we can understand that mariner is one kind of control system to keep safe navigation, which is operating the function of information processing based on the obtained information in navigational environment.

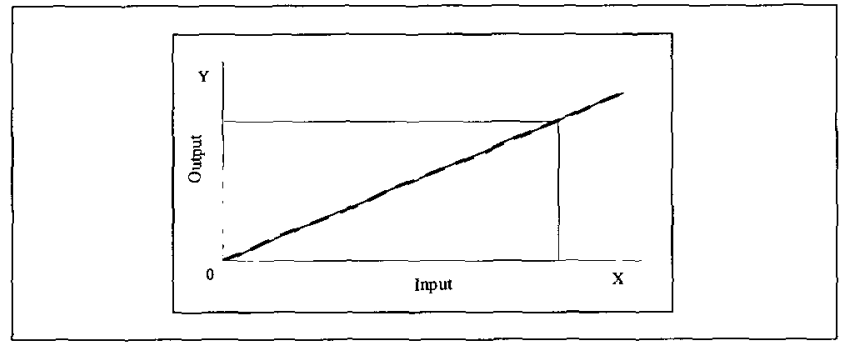

Fig. 1 The Relation between Input and Output in Mechanical System

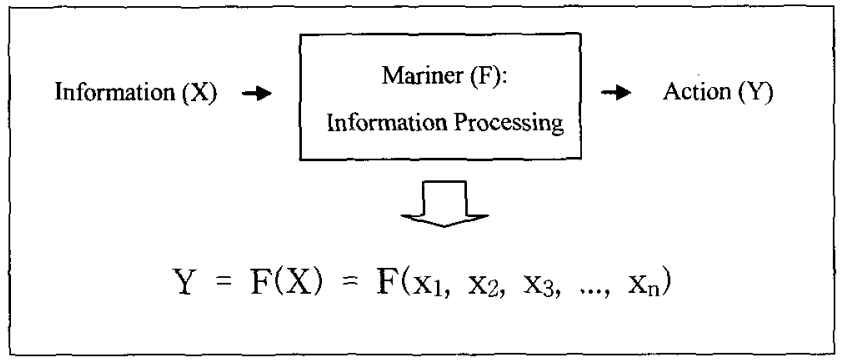

Fig. 2 Simple Model of Mariner's Behavior

'That is, mariner's avoiding behavior can be formulated based on the relation of three factors that are one mission to attain, mission-related information processing and necessary information as explained above.

\section{Modeling of Standard Avoiding Behavior}

In this section, the standard characteristics of mariner's behavior are analyzed by applying the data from the collaborative research on human factors in ship handling (Kobayashi, 2005b) and the model on the mariner's recognition process of dangerous ship for collision avoidance is introduced by using regression analysis.

\subsection{Mariner's Behavior in Recognition Process}

The recognition process can be expressed by the relation between one mission $\left(\mathrm{M}_{\mathrm{R}}\right)$, mariner's information processing (F) and necessary information (I). In other words, mariner's recognition to a dangerous ship $\left(\mathrm{M}_{\mathrm{R}}\right)$ is resulted from their information processing (F) based on necessary information (I: $I_{1}, I_{2}, I_{3}, \ldots, I_{n}$ ) as shown in Fig. 3 .

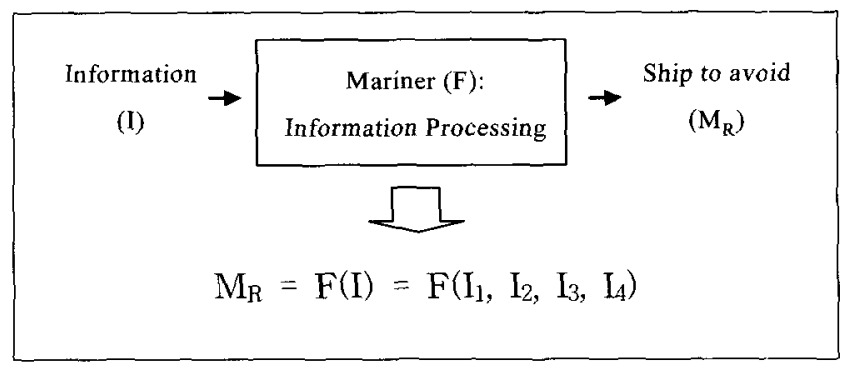

Fig. 3 Simple Model of Recognition Process

The detailed contents of one mission, the mission-related information processing and necessary information in recognition process are as follows (Park et al., 2007).

\section{$<$ Mission $>$}

$\mathrm{M}_{R}$ : To recognize a target vessel as a one required for avoiding actions.

$<$ Task>

$\mathrm{T}_{1}$ : To analyze the collision probability between the own ship and target ship.

$\mathrm{T}_{2}$; To confirm the interference condition to keep in mind if any avoiding action for the target ship is necessary.

$\mathrm{T}_{3}$; To analyze the surroundings.

$<$ Judgment>

$\mathrm{J}_{1}$ : To decide whether avoiding action is necessary or not. $\mathrm{J}_{2}$ : To decide whether there is anything else to analyze for the necessity of avoiding actions in surroundings or not.

$\mathrm{J}_{3}$ : To decide whether there is a ship to avoid or not.

$J_{4}$ : To decide whether the number of ship to avoid is plural or not.

$<$ Relating information $>$

$\mathrm{I}_{1}$ : Current interference condition

$\mathrm{I}_{2}:$ Estimated passing condition

$\mathrm{I}_{3}$ : Ship maneuverability

$\mathrm{I}_{4}$ : Traffic condition

In the contents of relating information, the current interference condition $I_{1}$ means the relation between an own ship and target ship, when they are underway and crossing, head on or overtaking situation. This condition can be expressed by factors such as courses and speeds of the own ship and the target ship, relative distances and bearings between the ships, and crossing angle of them. The estimated passing condition $\mathrm{I}_{2}$ can be explained by the distance of closest point of approach between the own ship 
and the target ship, the time to the closest point of approach between them, the passing distance of the target ship on the own ship's bow or stern line and the passing side of the target ship on the own ship's bow or stern line. The ship maneuverability $I_{3}$ means the ship's moment in general; however, it is estimated from the length of the ship in this paper. The traffic condition $\mathbf{I}_{4}$ means the relations among the own ship and the vessels surrounding the own ship, which are recognized by the officer on watch. The relation between the own ship and the target ship is not included in the traffic condition $\mathrm{I}_{4}$. The relating information $\mathrm{I}_{1} \sim \mathrm{I}_{4}$, therefore, can be defined as following sets;

$\mathrm{I}_{1}=\left\{\mathrm{Co}_{1}, \mathrm{Co}_{2}, \mathrm{~V}_{1}, \mathrm{~V}_{2}, \mathrm{a}, \Theta\right\}$

$\mathrm{I}_{2}=\left\{\right.$ TCPA, DCPA, $\left.\mathrm{P}_{\mathrm{DIS}}, \mathrm{P}_{\mathrm{DIR}}\right\}$

$\mathrm{I}_{3}=\left\{\mathrm{L}_{1}, \mathrm{~L}_{2}\right\}$

$\mathrm{I}_{4}=\left\{\right.$ COSUR, $\left.V_{\text {SUR }}, a_{\text {SUR }}, D_{\text {SUR }}, \Theta_{\text {SUR }}\right\}$

where, Col $\mathrm{Co}_{1}$ own ship's course, $\mathrm{V}_{1}$ : own ship's speed, $\mathrm{Co}_{2}$ : target ship's course, $\mathrm{V}_{2}$ : target ship's speed, $a$ : relative bearing, $\mathrm{D}_{\mathrm{R}}$ : relative distance,

$\Theta$ : crossing angle, $V_{R}$ : relative speed,

$\mathrm{P}_{\mathrm{DIS}}$ : passing distance, $\mathrm{P}_{\mathrm{DIR}}$ : passing direction, $\mathrm{L}_{1}$ : own ship's length, $\mathrm{L}_{2}$ : target ship's length, CoSUR, $V_{\text {SUR, }} a_{\text {SUR, }} D_{\text {SUR, }} \theta_{\text {SUR: }}$ courses, speeds, relative bearings, relative distances, crossing angles of the vessels surrounding own ship respectively.

In general the tasks $T_{1}$ and $T_{2}$, the collision probability between an own ship and a target ship and the necessity of avoiding action, can be determined by the current interference condition, estimated passing condition and ship maneuverability. The task $T_{3}$, analysis of surrounding situations, can be derived directly by the relative conditions of the surrounding vessels to an own ship. These relations between information and tasks can be expressed by following equations;

$$
\begin{aligned}
\mathrm{T}_{1} & =\mathrm{F}\left(\mathrm{I}_{1}, \mathrm{I}_{2}, \mathrm{I}_{3}\right) \\
\mathrm{T}_{2} & =\mathrm{F}\left(\mathrm{I}_{1}, \mathrm{I}_{2}, \mathrm{I}_{3}\right) \\
\mathrm{T}_{3} & =\mathrm{F}\left(\mathrm{I}_{4}\right)
\end{aligned}
$$

Using the equations (3), the judgements $\mathrm{J} 1^{\sim} \mathrm{J} 4$ can be expressed as follows;

$$
\begin{array}{ll}
\mathrm{J}_{1}=\mathrm{F}\left(\mathrm{T}_{1}\right) \\
\mathrm{J}_{2}=\mathrm{F}\left(\mathrm{J}_{1}, \mathrm{~T}_{2}, \mathrm{~T}_{3}\right) \\
\mathrm{J}_{3}=\mathrm{F}\left(\mathrm{J}_{1}, \mathrm{~T}_{2}, \mathrm{~T}_{3}\right)
\end{array}
$$

$\mathrm{J}_{4}=\mathrm{F}\left(\mathrm{J}_{1}, \mathrm{~T}_{2}, \mathrm{~T}_{3}\right)$

Consequently, the mission $\left(\mathrm{M}_{\mathrm{R}}\right)$ of the recognition process is resulted from the function of following equation,

$M_{R}=F\left(T_{1}, T_{2}, T_{3}, J_{1}, J_{2}, J_{3}, J_{4}\right)$

Therefore, we can understand that mariner's behavior in recognition process is decided by information processing based on the information.

\subsection{Application of the Model of Standard Behavior}

(1) Background and condition

The importance of study on human factors in ships has been increasing in all over the world. IMO proposed the importance of discussion on human factors. Thus we carried out the measurement on mariner's behavior during the training using a full-mission ship handling simulator in 8 institutes of 5 countries to understand mariner's behavior in important situation. We selected the situation in ship handling for collision avoidance as the important situation because the most of collisions are caused by human behavior. The navigational condition of scenario used in each training is different. Each institute measured important mariner's behavior in the case of handling for collision avoidance and we applied the obtained data to discuss the modeling method on the mariners' avoiding behavior. In this study, we dealt with the data when an own ship is a give-way vessel in crossing situation.

(2) The analysis items

We discussed the important behaviors of mariners for collision avoidance and the relating factors to influence on the their behavior to keep safety. The important mariner's behavior for collision avoidance is as follows;

- The first detection of target vessel

- The first recognition of target vessel considered as a danger

- The situation at the start point of avoiding action for collision avoidance

- The situation at the closest point of approach

- The situation at the position when a target ship pass an own ship's bow or stern line

And the evaluating factors of mariner's behavior are as follows;

(4) - Own ship's length (L)

- Own ship's speed $\left(V_{1}\right)$ 
- Target ship's speed $\left(\mathrm{V}_{2}\right)$

- Crossing angle $(\Theta)$

- Passing Distance (Pd)

- Time to the Closest Point of Approach (TCPA)

- Distance of the Closest Point of Approach (DCPA)

(3) Modeling of mariner's behavior in recognition process

The mariner's behavior in recognition process is discussed by applying the obtained data from the collaborative research mentioned above. The influence of relating factors to mariner's decisions in the recognition of dangerous ship for collision avoidance is analyzed. The equation on the relation between mariner's behavior and related factors decided by the regression analysis to model the recognition process is explained.

Td : Time to CPA at the first detection point of the target's existence

Tr : Time to CPA at the first recognition point of the danger to the target ship

Ta : Time to CPA at the start point of avoiding action to the target ship

Pd : Distance to the target ship at the position when the target ship passes an own ship's bow or stern line

(i) The relation between $\mathrm{Td}, \operatorname{Tr}$ and $\Theta$

Fig. 4 shows the relation between the detection time of target ships and the recognition time as dangerous ships using the information of TCPA. The determination coefficient $\left(R^{2}\right)$ and the correlation coefficient $(R)$ between the detection and recognition points is 0.4812 and 0.6937 respectively.

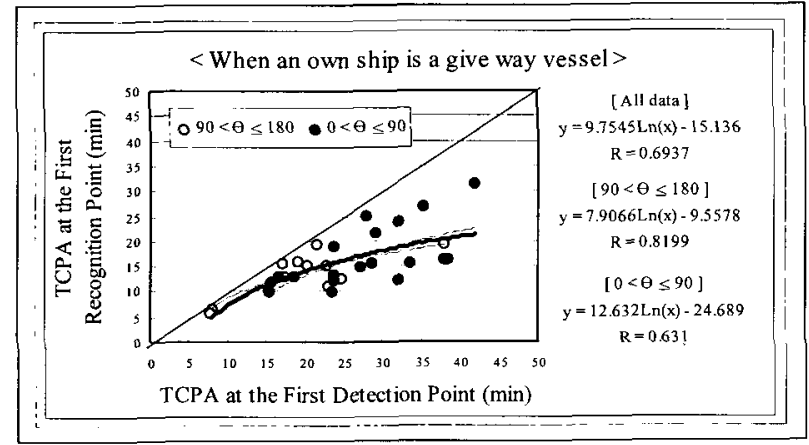

Fig. 4 Relation between Detection Points, Recognition Points and Crossing Angle (min-min)

The solid line of 45 degrees means the recognition and the detection is done at the same time. When the detection timing becomes later, the recognition becomes closer to the line of 45 degrees incline. On the contrary, when the detection timing is earlier, the recognition points separate from the line. That is, the relation shows that when the detection becomes later, mariners recognize the dangerous ship as soon as they detect the existence of target ship and when the detection is earlier, the time gap of recognition to detection becomes bigger. And the recognition poirts of target vessels with small crossing angle and big crossing angle shows almost the same tendency. Thus we can understand that the mariner's recognition to the dangerous ship depends on the timing of detection points and the relation between them is independent of the crossing angle. The equation (6) on the relation between the detection time of target ships and the recognition time as dangerous ships is given according to the Fig. 4 .

$$
\operatorname{Tr}=9.7545 \times \operatorname{Ln}(\mathrm{Td})-15.136
$$

(ii) The relation between $\operatorname{Tr}$, Ta and $\theta$

Fig. 5 shows the relation between recognition points, action points and crossing angle using the information of TCPA. The determination coefficient $\left(\mathrm{R}^{2}\right)$ and the correlation coefficient $(\mathrm{R})$ between the recognition and action points is 0.4486 and 0.6698 respectively.

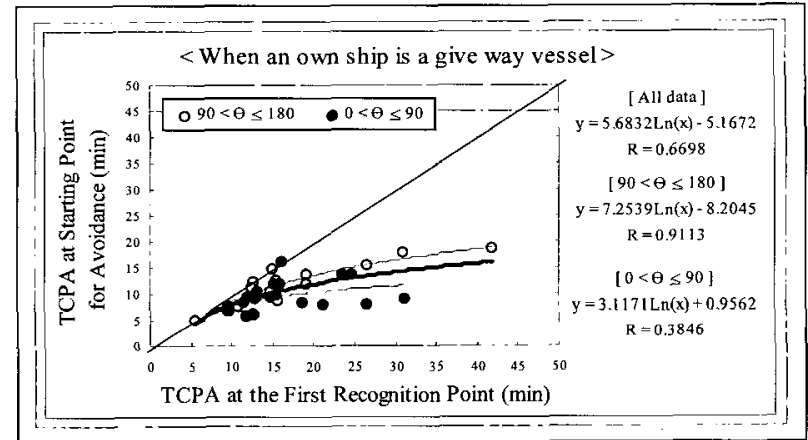

Fig. 5 Relation between Recognition Points, Action points and Crossing Angle ( $\min -\mathrm{min})$

The timing of recognition points has correlations with the one of action points. It is considered that the mariner's recognition timing of ships considered as danger has an influence on the decision of start timing for avoiding actions. Also, it is shown that the value of crossing angle makes different start timing for avoiding at the same timing of recognition points. That is, mariners start to avoid the target ships approaching with big crossing angle in earlier timing than those with small crossing angle. We can understand that the crossing angle between ships is one of important factors to affect mariner's behavior for avoiding actions. The equation (7) on the relation between the recognition time as dangerous ship and the start time of the avoiding action is given according to Fig. 5 . 
$\mathrm{Ta}=5.6832 \times \operatorname{Ln}(\mathrm{Tr})-5.1672$

(iii) The relation between Ta, DCPA, $\theta$ and $\mathrm{L}$

Fig. 6 shows the relation between DCPA, starting points of avoiding action and crossing angle. The determination coefficient $\left(\mathrm{R}^{2}\right)$ and the correlation coefficient $(R)$ between the start timing of avoiding action and DCPA/L is 0.18 and 0.4243 respectively.

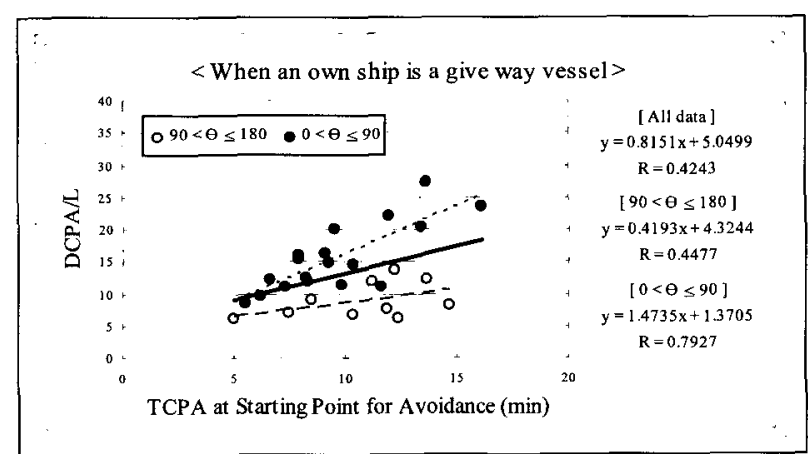

Fig. 6 Relation between DCPA, Starting Points for Avoiding and Crossing Angle (NM/L-min)

The DCPA/L is the ratio of DCPA to the own ship's length $(\mathrm{L})$, which means the distance of the closest point to the target ship in related to the own ship's length. The relation shows that earlier avoiding action and larger size of the own ship can make the DCPA bigger. And also, there is a tendency that mariners keep the distance of CPA to the target ship that is, at least, more than 5 times of the own ship's length. That is, the timing of action points has correlations with the distance of CPA in related to the own ship's size.

Furthermore, as shown in Fig. 6, the value of crossing angle makes different DCPA/L at the same start timing of action points. In encountering situation that the crossing angle between the ships is big, DCPA $/ \mathrm{L}$ is distributed in smaller value than the ones that the crossing angle is small. That is, mariners trend to keep smaller distances to the target ships encountering with big crossing angle than the ones with small crossing angle. Thus we can understand that mariner's start timing of avoiding action has an influence on the results of DCPA, and the own ship's size and the crossing angle are the important factors to affect mariner's behavior in deciding the distance of CPA to the target ship. The equation (8) and (9) on the relations between the DCPA, the start time of the avoiding action and the own ship's length are given according to Fig. 6 respectively.
DCPA $>5 \times \mathrm{L}$

(iv) The relation between $\mathrm{Ta}, \mathrm{Pd}, \Theta$ and $\mathrm{L}$

Fig. 7 shows the relation between passing distance, start points of avoiding action and crossing angle. The determination coefficient $\left(\mathrm{R}^{2}\right)$ and the correlation coefficient (R) between the start timing of avoiding action and Passing Distance/L is 0.2678 and 0.5175 respectively.

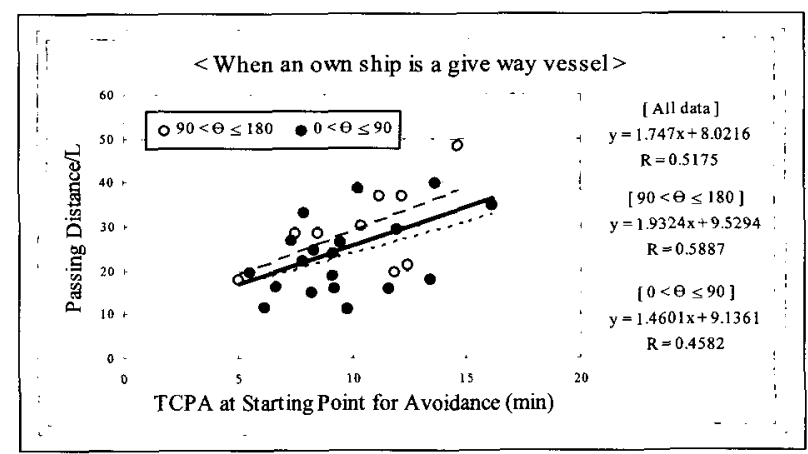

Fig. 7 Relation between Passing Distance, Starting Points for Avoiding and Crossing Angle (NM/L-min)

Passing Distance/L is the ratio of passing distance to the own ship's length(L), which means the distance of target ship to the own ship at the position where the target ship passes the own ship's bow line. The relation shows that earlier avoiding action and larger size of an own ship can make Passing Distance bigger. That is, the start timing of action points has correlations with the passing distance in related to the own ship's length. And also, there is a tendency that mariners keep the passing distance to the target ship that is, at least, more than 10 times of the own ship's length. Furthermore, as shown in Fig. 7, the value of crossing angle makes different Passing Distance/L at the same start timing of action points. In encountering situation that the crossing angle between the ships is big, Passing Distance/ $L$ is distributed in bigger value than the ones that the crossing angle is small. That is, mariners trend to keep bigger passing distances to the target ships encountering with big crossing angle than the ones with small crossing angle. Thus we can understand that mariner's start timing of avoiding action has an influence on the results of passing distance, and the own ship's size and crossing angle are the important factors to affect mariner's behavior in deciding passing distance to the target ship. The equation (10) and (11) on the relations between the passing distance, the start timing of the avoiding action and the own ship's length is given according to Fig. 7 respectively.

$$
\mathrm{Pd}=(1.747 \times \mathrm{Ta}+8.0216) \times \mathrm{L}
$$


$\mathrm{Pd}>10 \times \mathrm{L}$

As shown in equation (9) and (11), we can consider that mariners measure the collision probability with the DCPA and the passing distance in related to the own ship's length. That is, when the distance of CPA is less than 5 times of the own ship's length and the distance to the target ship passing in the own ship's bow line is less than 10 times of the own ship's length, mariners recognize the collision probability between the own and target ship. Thus we can define the distance of CPA of 5 times and passing distance of 10 times to the own ship's length as "safety distance" to keep between ships in the situation of collision avoidance. The equation (12) on the relation between the recognition time and the distance of CPA can be calculated by equation (7), (8) and (9).

$$
\begin{aligned}
& D C P A=\{0.8151 \times(5.6832 \times \operatorname{Ln}(\operatorname{Tr})-5.1672)+5.0499\}>5 \\
& \operatorname{Ln}(T r)>0.898, \quad \therefore \operatorname{Tr} \geq 2.45
\end{aligned}
$$

And the equation (13) on the relation between the recognition time and the passing distance can be calculated by equation (7), (10) and (11).

$$
\begin{aligned}
& \operatorname{Pd}=\{1.747 \times(5.6832 \times \operatorname{Ln}(\operatorname{Tr})-5.1672)+8.0216\}>10 \\
& \operatorname{Ln}(\operatorname{Tr})>1.01, \quad \therefore \operatorname{Tr} \geq 2.75
\end{aligned}
$$

Thus the equation (14) on the recognition timing can be expressed by equation (6), (12) and (13).

$$
\therefore 2.75 \leq \mathrm{Tr}<\mathrm{Td}
$$

As shown in equation (14), therefore, we confirmed that mariners should recognize the target ships in earlier timing than, at least, 2.75 minutes to the closest distance after detecting the target ship in order to keep the closest distance of 5 times and the passing distance of 10 times to the own ship's length.

As a result, we can conclude that mariners generally trend to recognize the target ship as a danger in earlier timing than 5 minutes to the closest point, but they need to recognize the dangerous target ship, at least, before 2.75 minutes to the closest point in order to keep the safety distance when we consider that the recognition and avoiding action can be taken simultaneously in late point of recognition as shown in Fig. 5.

\subsection{Summary}

(i) The mariner's recognition of the dangerous ship depends on the timing of detection points and the relation between them is independent of crossing angle.

(ii) The mariner's start timing of avoiding action depends on the timing of recognition points and the relation between them is affected by crossing angle.

(iii) The distance of CPA and passing distance depend on the start timing of avoiding action and the relation between them is affected by the own ship's size and crossing angle.

(iv) The mariner's recognition to the target ship as a danger depends on the detection timing and mariners recognize the target ship as a danger when the distance of CPA to the target ship is less than 5 times of the own ship's length and the distance passing in the own ship's bow line is less than 10 times of the own ship's length. And also, mariners need to recognize the target ship in earlier timing than, at least, 2.75 minutes to CPA in order to keep the distance of the closest point of approach of 5 times and the passing distance of 10 times to the own ship's length after detecting the existence of target ships.

\section{Conclusion}

We proposed the modeling method on mariner's avoiding behavior with an example of recognition process by applying the results of the collaborative research on human factors in ship handling in this paper. As a result, the obtained results are as follows.

(i) The standard characteristics of mariner's behavior in recognition process for collision avoidance are clarified.

(ii) The relation between mariner's behavior and the related factors are analyzed.

(iii) The modeling method of mariner's behavior for collision avoidance based on human factors are proposed.

(iv) The model on mariner's recognition behavior is introduced by using regression analysis.

The completed model of mariners' standard behavior for collision avoidance will be dealt in future study.

\section{References}

[1] Kobayashi, H. (2003), "Simulator Application", Proceeding of International Conference on Marine Simulation and Ship Maneuverability, pp. 1-12.

[2] Kobayashi, H. (2004), "Application of Maritime Simulator for Enhancement of Safety Navigation", Proceeding of 4th Japan-Korea Workshop on Marine Simulator Research, pp. 1-13.

[3] Kobayashi, H. (2005a), "Functional Approach on the Techniques of Ship Handling", Proceeding of 5th Asian 
Conference on Marine Simulator and Simulation Research, pp. 1-10.

[4] Kobayashi, H. (2005b), "Report of international collaborative research on human factor in ship handling", International Marine Simulator Forum, Memorial University of Newfoundland, Canada.
[5] Park, J. S., Kobayashi, H. and Yea, B. D. (2003), "The Relation between Human Behavior and Safety in the Collision Avoidance Situation", Journal of Korean Navigation and Port Research, Vol.27, No.6, pp. 611-618.

[6] Park, J. S., Kobayashi, H. and Yea, B. D. (2007), “A study on Mariners' Standard Behavior (1)", Proceeding of Korean Navigation and Port Research, pp. 29-35.

Received 6 April 2007

Accepted 30 June 2007 